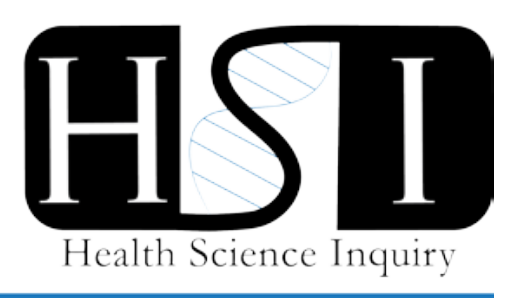

\title{
Diet as a modifiable contributor and potential treatment strategy for Major Depressive Disorder
}

\author{
Caroline Wallace \\ Department of Psychiatry, Queen's University
}

\section{Top Submission}

Much like the recent push in medicine to have physicians consider mental health when treating physical illness, a similar drive is needed for the inverse to occur in psychiatry: to consider physical health when treating mental illness. A novel and promising avenue for this is monitoring and modifying diet. Typically recognized as a critical factor in physical health, diet is a controllable health behaviour that has been associated with mental health resulting from an increased understanding of the interactions between the gastrointestinal tract and the brain [1]. These interactions occur through a bi-directional communication network, aptly termed as the gut-brain axis. This area of study has seen a proliferation in preclinical, clinical, and translational research, and has implicated both diet and nutrition as modifiable contributors to chronic mental illnesses, such as Major Depressive Disorder (MDD) [2].

MDD (also known as clinical depression) is a serious, and often chronic, psychiatric condition that affects approximately $11 \%$ of Canadians at some point in their lifetime [3]. Having no clear etiology, MDD is often treated with antidepressant medications that alter neurotransmitter activity in the brain. However, these medications simply treat the symptoms of depression, but not the disorder, itself. With numerous complications arising from the use of antidepressants, focus has now shifted to treatments that target the possible underlying pathophysiology of the disorder.

A consistent finding among patients with depression, and a proposed cause of the disorder, is an increased expression of pro-inflammatory cytokines that lead to neuroinflammation. Once in the brain, pro-inflammatory cytokines reduce the availability of neurotransmitters that are critical for regulating mood, such as serotonin, dopamine, and norepinephrine, by reducing their synthesis [4]. They also reduce neurotransmitter availability by increasing the expression of transporters that reabsorb them into the presynaptic neuron, and by decreasing precursors of these neurotransmitters by activating enzymes that break them down [4]. These alterations in neurotransmitter functioning are associated with changes in reward circuity in the prefrontal cortex and the anterior cingulate cortex, as well as with fear and arousal circuitry in the amygdala and the insula, which manifest as the core features of depression, such as anhedonia and anxiety [4]. Research on the gut-brain axis has helped researchers hypothesize the etiology of this immune activation, and has emerged as a possible treatment target for MDD.

Chronic stress, often a precursor for depression, can cause increased permeability of the gastrointestinal lining, and allow toxins, such as the endotoxin Lipopolysaccharide (LPS), to leak into the bloodstream [5]. LPS is known to elicit a strong global immune response, including a release of pro-inflammatory cytokines. These cytokines can then travel to the brain, cause disruptions in neurotransmission, as described above, and result in a depressive symptomatology [4]. Thus, it is hypothesized that targeting the gut-brain axis and reducing inflammation may alleviate the symptoms of depression.

One of the most direct ways to target the gut-brain axis is through an individual's nutrient intake. While research studying the effects of diet and nutrition on MDD in clinical samples is still in its early stages, several studies have reported robust findings that support the idea that specific nutrient-and diet-based interventions can alleviate depressive symptoms. Nutrients that may be contributing to this effect include polyunsaturated fatty acids (PUFAs), such as omega- 3 and omega- 6 , Vitamin D, and probiotics. A recent review by Deacon and colleagues reported that there is adequate evidence for suggesting that omega$3 s$ have a role in improving depression, but that further research is required [6]. Studies assessing the effects of probiotics on alleviating the symptoms of depression and anxiety in humans also show promise, but there currently exist too many gaps and inconsistencies in the research, as well as a lack of randomized controlled trials from which to draw conclusions [7].

From a whole-diet perspective, dietary patterns that are high in plant-based foods and lean proteins, and that 
are based on consuming fresh and minimally processed foods, such as the Mediterranean diet, have garnered attention in the field of nutritional psychiatry for improving mental health. Jacka and colleagues recently conducted a randomized controlled trial (SMILES trial) and showed that a modified Mediterranean diet support group had a greater improvement in the symptoms of depression in clinically depressed individuals than a social support group [8]. Parletta and colleagues also conducted a trial (HELIFMED) comparing a Mediterranean-style dietary intervention and a social support condition as a control, and found similar results [9]. Unsurprisingly, the Mediterranean diet is rich in nutrients that are potentially linked to improved mental health, and that have been consistently associated with lower levels of inflammation.

Although the research to date that directly implicates nutrient intake and diet with improved symptoms of depression is by no means conclusive, it may eventually present an enticing alternative treatment option for MDD. Improving nutrient intake by modifying diet is an accessible lifestyle change with no adverse side effects and may translate to improved mental health. These lifestyle changes have considerable implications for individuals who are unable or do not wish to take antidepressant medication. Moreover, individuals who maintain a healthy diet are more likely to engage in other protective health behaviours that may also help to improve mental health. For these reasons, research on the impact of diet on MDD should focus on clinical populations, and should be considered in clinical settings for the treatment of mental illness.

\section{References}

[1] Foster, J. A., Neufeld, K. A. (2013) Gutbrain axis: how the microbiome influences anxiety and depression. Trends in neurosciences, 36 (5), 305-312.
[2] Dinan, T. G., Quigley, E. M. (2011) Probiotics in the treatment of depression: science or science fiction?. Aust N Z J Psychiatry, 45 (12), 1023-1025.

[3] Pearson, C., Janz, T., Ali, J. (2013) Mental and substance use disorders in Canada. Statistics Canada Statistics Canada Catalogue no. 82-624-X.

[4] Miller, A. H., Raison, C. L. (2016) The role of inflammation in depression: from evolutionary imperative to modern treatment target. Nature Reviews Immunology, 16 (1), 22.

[5] Kelly, J. R., Kennedy, P. J., Cryan, J. F., Dinan, T. G., Clarke, G., Hyland, N. P. (2015) Breaking down the barriers: the gut microbiome, intestinal permeability and stress-related psychiatric disorders. Frontiers in cellular neuroscience, 14 (9), 392.

[6] Deacon, G., Kettle, C., Hayes, D., Dennis, C., Tucci, J. (2017) Omega 3 polyunsaturated fatty acids and the treatment of depression. Critical reviews in food science and nutrition, 57 (1), 212-223.

[7] Wallace, C. J. K., Milev, R. (2016) The effects of probiotics on depressive symptoms in humans: a systematic review. Annals of general psychiatry, 6 (1), 14.

[8] Jacka, F. N., O'Neil, A., Opie, R., Itsiopoulos, C., Cotton, S., Mohebbi, M., Castle, D., Dash, S., Mihalopoulos, C., Chatterton, M. L., Brazionis, L. (2017) A randomised controlled trial of dietary improvement for adults with major depression (the SMILES'trial). BMC medicine, 15 (1), 23.

[9] Parletta, N., Zarnowiecki, D., Cho, J., Wilson, A., Bogomolova, S., Villani, A., Itsiopoulos, C., Niyonsenga, T., Blunden, S., Meyer, B., Segal, L. (2017) A Mediterranean-style dietary intervention supplemented with fish oil improves diet quality and mental health in people with depression: A randomized controlled trial (HELFIMED). Nutritional neuroscience, 8, 1-4.

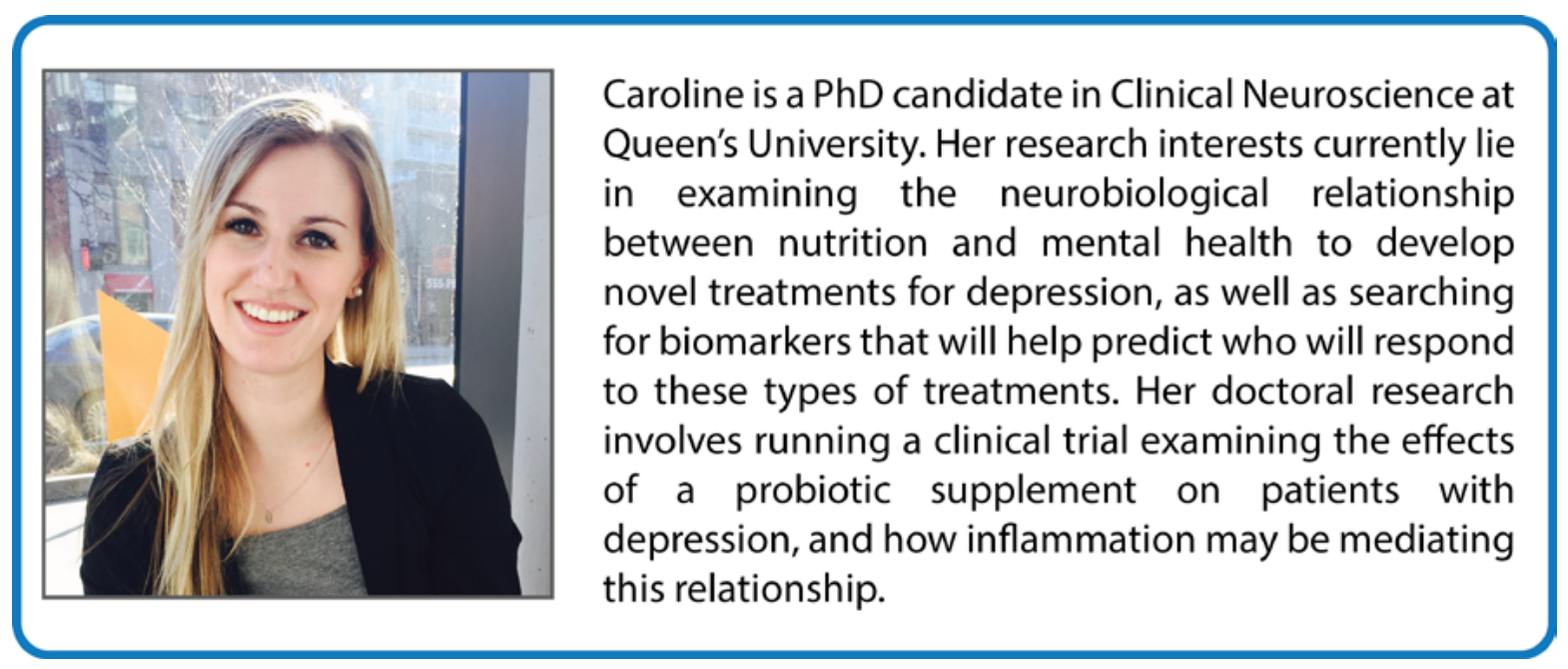

